Open Access

\title{
A Case of Gossypiboma Masquerading as a Gastrointestinal Stromal Tumor
}

\author{
Jong Woon Cheon', Eun Young Kim¹, Ki Yong Kim¹, Jae Bum Park¹, \\ Young Kook Shin ${ }^{1}$, Ka Young Kim ${ }^{1}$ and Hyun Dong Chae ${ }^{2}$ \\ Departments of ${ }^{1}$ Internal Medicine and ${ }^{2}$ Surgery, Catholic University of Daegu College of Medicine, Daegu, Korea
}

\begin{abstract}
Gossypiboma refers to a mass resulting from a retained gauze pad accidentally left within the body after surgery. Although the clinical features are diverse, it is often found incidentally as a mass having an internal cystic change and adhesion to adjacent organs. Abdominal computed tomography (CT) is helpful, yet the initial diagnosis can be misleading in cases with atypical findings. We report a case of gossypiboma in a 78-year-old woman that we suspected was a gastrointestinal stromal tumor according to abdominal CT and endoscopic ultrasound, yet was diagnosed as a gossypiboma postoperatively.
\end{abstract}

Key Words: Gossypiboma; Endoscopic ultrasonography; Gastrointestinal stromal tumor

\section{INTRODUCTION}

Gossypiboma refers to a mass resulting from retained cotton, gauze, or sponge accidentally left within the body after surgery. As indicated in its name, gossypiboma is an iatrogenic outcome reported on rare occasions in various parts of the body with diverse clinical findings, often mistaken radiologically as a tumor or abscess. Now, we report a literature review and our experience of a case with incidentally found mass, who was first judged as a gastrointestinal stromal tumor (GIST) of the gastric fundus by abdominal computed tomography (CT) and endoscopic ultrasonography (EUS), but was later confirmed as gossypiboma resulting from retained surgical sponge.

\section{CASE REPORT}

A 78-year-old female visited the hospital for sudden onset epigastric pain which lasted for one day. The pain developed

Received: April 9, 2011 Revised: August 25, 2011

Accepted: August 31, 2011

Correspondence: Eun Young Kim

Department of Internal Medicine, Catholic University of Daegu College of Medicine, 3056-6 Daemyeong 4-dong, Nam-gu, Daegu 705-718, Korea Tel: +82-53-650-4092, Fax: +82-53-628-4005, E-mail: kimey@cu.ac.kr

(a) This is an Open Access article distributed under the terms of the Creative Commons Attribution Non-Commercial License (http://creativecommons.org/ licenses/by-nc/3.0) which permits unrestricted non-commercial use, distribution, and reproduction in any medium, provided the original work is properly cited. around midnight before the visit, accompanying nausea and vomiting. She received abdominal US at a private clinic indicating multiple gallbladder (GB) stones and common bile duct (CBD) stones and was transferred to the emergency room of this hospital. She had a history of partial gastrectomy 30 years ago in another hospital for a reason she cannot remember precisely. The physical examination revealed blood pressure 110/70 $\mathrm{mm} \mathrm{Hg}$, pulse rate $76 / \mathrm{min}$, respiration rate $20 / \mathrm{min}$, and body temperature $36.6^{\circ} \mathrm{C}$ without unusual finding except for the epigastric tenderness. Peripheral blood test showed hemoglobin $9.2 \mathrm{~g} / \mathrm{dL}$, white blood cell (WBC) $9,600 / \mathrm{mm}^{3}$, and platelet $428,000 / \mathrm{mm}^{3}$. Biochemistry analysis showed AST $259 \mathrm{IU} / \mathrm{L}$, ALT $95 \mathrm{IU} / \mathrm{L}$, total bilirubin $1.5 \mathrm{mg} / \mathrm{dL}$, alkaline phosphatase $404 \mathrm{IU} / \mathrm{L}, \gamma$-GTP $58 \mathrm{IU} / \mathrm{L}$, amylase $66 \mathrm{U} / \mathrm{L}$, and lipase $26 \mathrm{U} / \mathrm{L}$. The abdominal CT performed at the emergency room indicated mild dilatation of intrahepatic bile duct and CBD with multiple GB stones, but the finding of CBD stone was not definite. In addition, a 5.5-cm sized well-demarcated round mass with irregular calcification density was found adjacent to the gastric fundus (Fig. 1). Esophagogastroduodenoscopy (EGD) was performed to search the cause of the abdominal pain, where 5-cm sized bulging area covered with normal mucosa was found at the gastric fundus but not any other lesions to cause the abdominal pain (Fig. 2). CBD stone was not detectable on EUS performed to exclude the presence of CBD stone. EUS also showed an exophytic hypoechoic mass which was connect- 
ed with the fourth layer of the gastric wall at the area where subepithelial tumor was suspected. Hyperechoic area with posterior acoustic shadowing was observed inside the mass (Fig. 3). The above mentioned findings suggested GIST of the gastric fundus accompanying internal calcification. The symptoms improved after conservative treatment. Laboratory findings were normalized and spontaneously passed CBD stone was suspected. Later she underwent surgery for cholecystectomy and resection of the mass lesion. Upon laparotomy, a brownish hard mass was discovered between the left diaphragm and gastric fundus. It appeared, from the operative field, that the mass was a foreign body covered by the inflammatory membrane rather than a GIST of the gastric fundus. The mass was tightly adhered to the gastric fundus, making it difficult to dissect from the gastric wall. The mass was resected by wedge resection of the gastric fundus. The resected mass was $6.5 \times 4 \mathrm{~cm}$ in size and when the mass was cut in half, a surgical sponge was visible with the naked eyes (Fig. 4). The covering material was confirmed as inflammatory pseudomembrane accompa-

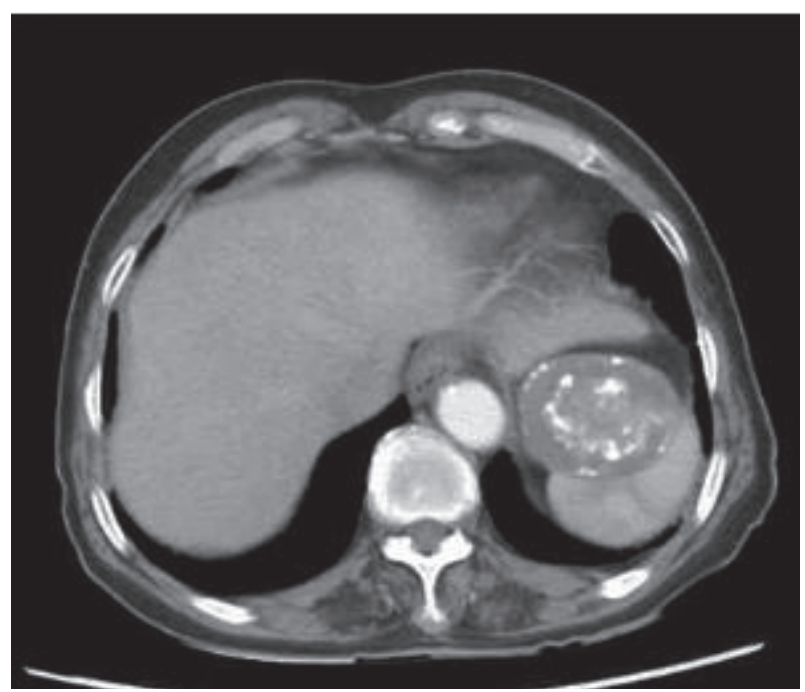

Fig. 1. Abdominal computed tomography finding. A well-circumscribed, $5.5 \mathrm{~cm}$-sized round mass with irregular internal calcification is noted in contiguity with the gastric fundus. nying fibrosis, abnormal calcification, hemorrhage, and necrosis.

\section{DISCUSSION}

Gossypiboma, also called 'textiloma', is a compound of 2 words "gosysspium" meaning 'cotton' in Latin and "boma" meaning 'place of concealment' in Swahili, referring to a mass resulted from the retained cotton or gauze after the operation. ${ }^{1}$ The occasion of retaining surgical instruments inside the human body is rare but there have been always the possibility from the beginning of the history of surgery. Every surgery with incisional wound has the potential to leave a foreign body inside the human body, such as gauze, clamp, retractor, or electrode. Surgical sponge, among others, is most frequently left in the human body after surgery, as much as to be named separately as gossypiboma or textiloma. Gossypiboma within the abdominal cavity in most patients are asymptomatic and often leads to a medicalmalpractice claim only by incidental discovery several years later,

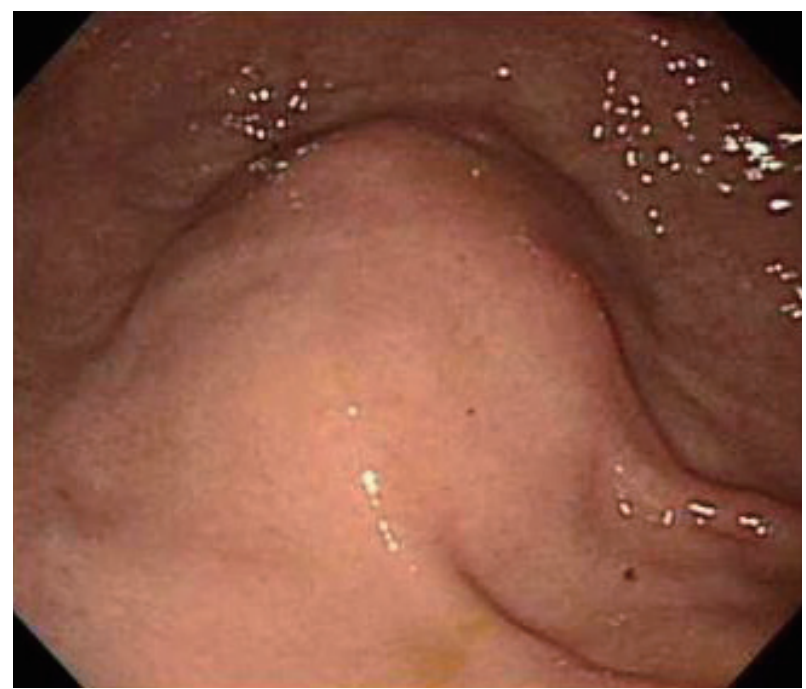

Fig. 2. EGD finding. A bulging lesion with normal overlying mucosa was seen at the fundus.
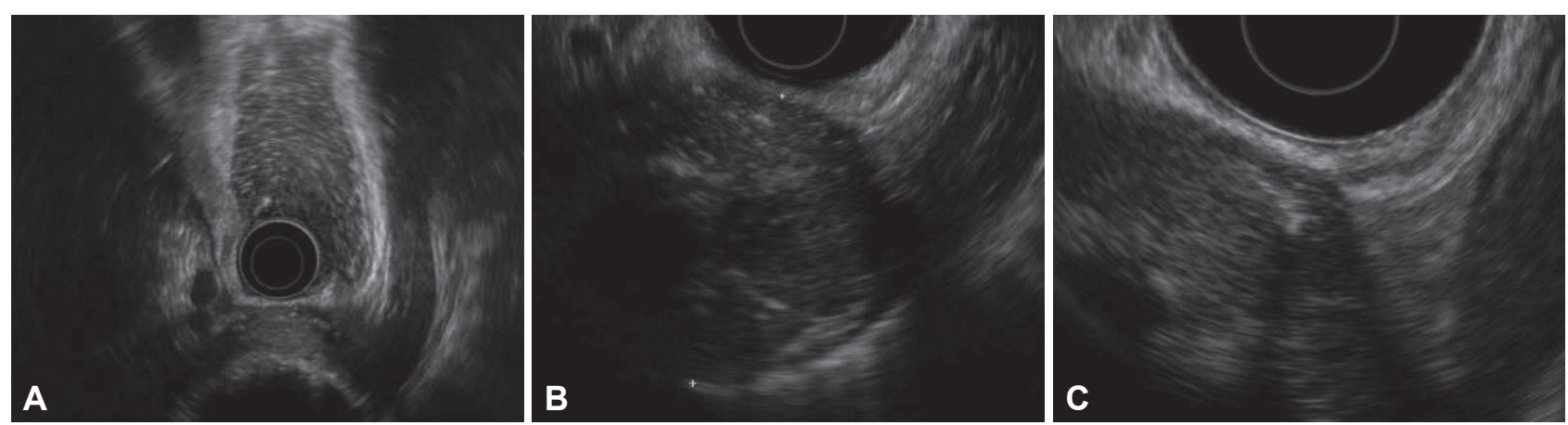

Fig. 3. Endoscopic ultrasonography findings. (A) An exophytic growing hypoechoic mass showing central linear hyperechoic area with posterior acoustic shadowing was noted. (B) Hypoechoic mass with internal irregular hyperechoic spots. (C) The mass is connected with fourth gastric wall layer and gastric serosal layer was not delineated. 

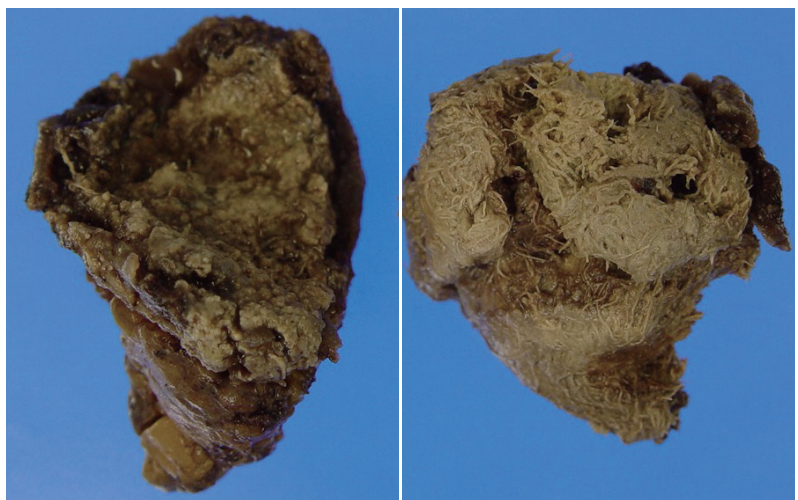

Fig. 4. Gross findings. The mass is found to be a retained surgical sponge, so-called 'gossypiboma' surrounded by inflammatory pseudomembranous tissue which is tightly attached to gastric wall.

further complicating the recognition of precise incidence. The incidence of gossypiboma in South Korea is unknown but reported about 1/1,000-10,000 surgeries worldwide. ${ }^{2}$ Gossypiboma complication might occur in every form of surgery, ranging from general surgery (52\%) to gynecology (22\%), to urology and vascular surgery (10\%), and to ophthalmology and spinal surgery (6\%). ${ }^{2}$ A case-control study by Gawande et al. ${ }^{3}$ found emergency operation, unexpected change in operation, and high body mass index of the patient were the risk factors of leaving a foreign material inside the human body after the operation.

Cotton surgical sponge does not cause any specific biochemical reaction except for the adhesion or granuloma formation inside the body. ${ }^{4}$ The clinical manifestation of gossypiboma depends on the type of reaction, the location of gauze or sponge, and the degree of chronicity, but could be separated into 2 groups. ${ }^{5}$ The first group is the exudative inflammatory reaction which could be detected early after the surgery by symptoms caused by the abscess formation, or could be detected later with intestinal obstruction, erosion into the adjacent organs, and fistula formation. The retained gauze inside the abdominal or pelvic cavity could erode into the gastrointestinal tract causing extracorporeal extrusion on rare occasions. The second group is the aseptic fibrinous reaction where the encapsulated sponge does not induce symptoms and would not be detected for a long time. Our patient belongs to the latter, since she did not have any symptom for as long as 30 years, and the gauze was encapsulated and adhered to the gastric fundus.

Abdominal CT is most effective and practical diagnostic method for gossypiboma. There are various CT findings but a cystic lesion with marked rim enhancement is most common. ${ }^{6}$ An investigator termed the calcium deposition around the rim of the retained surgical sponge appearing in the form of calcified reticulate rind as "calcified reticulate rind sign" and reported it as the characteristic CT finding of gossypiboma. Hypoechoic mass with strong posterior acoustic shadowing due to hyperechoic capsule suggests gossypiboma on the US. ${ }^{6}$ In this patient the mass showed irregular calcification at the center area of the mass. Every gauzes recently used for surgery contains radiopaque markers for easier detection by postoperative radiography. Gauzes in which these markers are not contained or degraded, or calcified may not reveal the characteristic radiopaque line on the radiography.

There is no specific EUS finding for gossypiboma. EUS could be performed for detailed observation when a gastrointestinal subepithelial lesion is suspected. In the patient of this study, it was considered that the $5 \mathrm{~cm}$ sized protruded area covered by normal mucosa on EGD was the same mass discovered on CT appearing to originate from the stomach. EUS was performed for more detailed observation, revealing a hypoechoic mass originating from the fourth gastric wall layer. It is presumed that the inflammatory mass encapsulating the gauze was adhered to the serosal layer due to the fibrosis, and appeared as hypoechoic on EUS. Subepithelial lesion on EUS is diagnosed putatively based on the layer of origination and echogenic findings of the mass. When the pathologic diagnosis and EUS impression of subepithelial lesions was compared in the prior studies, the diagnostic accuracy of EUS was estimated about $78 \% .{ }^{8}$ The factors contributing to the incorrect EUS interpretation include inflammatory change, fibrosis, or tumor microinvasion around the tumor. ${ }^{9}$ The typical EUS finding of GIST is hypoechoic tumor originating from the fourth (proper muscle) layer, occasionally in multilobulated or pedunculated forms. Findings of necrosis, calcification, or ulceration of GIST suggests the possibility of malignant transformation. ${ }^{10}$

Our case did not show spongiform air bubbles with marked rim enhancement and cystic lesion on CT as typically found in gossypiboma patients. But the round well-demarcated mass originated from the stomach accompanying radiopaque material was noted and mistaken as a mass with irregular calcified density. This lesion was also found on EUS as an exophytic hypoechoic mass connected with the fourth layer of the gastric wall and internal irregular hyperechoic spots with posterior acoustic shadowing. These findings suggested GIST of the gastric fundus with internal calcification requiring surgery. But the resected lesion was found to be a mass resulted from the retained surgical sponge.

\section{Conflicts of Interest}

The authors have no financial conflicts of interest.

\section{REFERENCES}

1. Lu YY, Cheung YC, Ko SF, Ng SH. Calcified reticulate rind sign: a characteristic feature of gossypiboma on computed tomography. World J Gastroenterol 2005;11:4927-4929.

2. Yamamura N, Nakajima K, Takahashi T, et al. Intra-abdominal textiloma. A retained surgical sponge mimicking a gastric gastrointestinal stromal tumor: report of a case. Surg Today 2008;38:552-554.

3. Gawande AA, Studdert DM, Orav EJ, Brennan TA, Zinner MJ. Risk fac- 
tors for retained instruments and sponges after surgery. N Engl J Med 2003;348:229-235.

4. Sturdy JH, Baird RM, Gerein AN. Surgical sponges: a cause of granuloma and adhesion formation. Ann Surg 1967;165:128-134.

5. Choi BI, Kim SH, Yu ES, Chung HS, Han MC, Kim CW. Retained surgical sponge: diagnosis with CT and sonography. AJR Am J Roentgenol 1988;150:1047-1050.

6. Kokubo T, Itai Y, Ohtomo K, Yoshikawa K, lio M, Atomi Y. Retained surgical sponges: CT and US appearance. Radiology 1987;165:415-418.

7. Kopka L, Fischer U, Gross AJ, Funke M, Oestmann JW, Grabbe E. CT of retained surgical sponges (textilomas): pitfalls in detection and evaluation. J Comput Assist Tomogr 1996;20:919-923.

8. Kwon JG, Kim EY, Kim YS, et al. Accuracy of endoscopic ultrasonographic impression compared with pathologic diagnosis in gastrointestinal submucosal tumors. Korean J Gastroenterol 2005;45:88-96.

9. Kim EY. Endoscopic ultrasonography in locoregional staging of gastric cancer. Korean J Gastroenterol 2008;52:124-127.

10. Darnell A, Dalmau E, Pericay C, et al. Gastrointestinal stromal tumors. Abdom Imaging 2006;31:387-399. 